\title{
Über Carboxylverbindungen der Diarylalkane und -alkene II ${ }^{1}$
}

\author{
$\beta, \beta$-Dichlor- $\alpha, \alpha$-bis-[4-carboxy-phenyl]-äthylen und verwandte Verbindungen \\ Von RANDOLPH RIEMSCHNEIDER ${ }^{2}$
}

(Z. Naturforschg. 6 b, 179-183 [1951]; eingegangen am 31. März 1951)

\begin{abstract}
$\beta, \beta$-Dichlor- $\alpha, \alpha$-bis-[4-carboxy-phenyl]-äthylen (VI) vom Schmp. 276-277 4 kann sowohl durch Oxydation von $\beta, \beta, \beta$-Trichlor- $\alpha, \alpha$-bis-[4-methyl-phenyl]-äthan als auch durch Umsetzung von $\beta, \beta, \beta$-Trichlor- $\alpha, \alpha$-diphenyl-äthan mit Oxalylchlorid in Gegenwart von Aluminiumchlorid erhalten werden. Seine Konstitution wurde durch Überführung in geeignete Derivate und Abbauversuche sichergestellt. Nebenprodukte bei der Synthese von VI sind die Säuren $\beta, \beta, \beta$ Trichlor- $\alpha$-[4-methyl-phenyl]- $\alpha$-[4-carboxy-phenyl]-äthan (VII) vom Schmp. $160^{\circ}$ bzw. $\beta, \beta$ Dichlor- $\alpha$-phenyl- $\alpha$-[4-carboxy-phenyl]-äthylen (XIV) vom Schmp. $203^{\circ}$. Die von früheren Untersuchern 3 als $\beta, \beta, \beta$-Trichlor- $\alpha, \alpha$-bis-[4-carboxy-phenyl]-äthan (III) und III-diamid (V) bezeichneten DDT-Analogen wurden als VI und $\beta, \beta$-Dichlor- $\alpha, \alpha$-bis-[4-carbamido-phenyl]äthylen (XI) erkannt.

Sämtliche der bisher im Petrischalen-Filmtest an Drosophila melanogaster M. und Musca domestica L. auf kontakt-insektizide Wirksamkeit geprüften Carboxylverbindungen der DDTReihe und ihrer Derivate haben sich als unwirksam erwiesen.
\end{abstract}

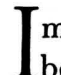
$m$ Rahmen von Untersuchungen über DDT-Derivate beschäftigten sich L. Haskelberg und D. Lavie ${ }^{3}$ mit der Frage, ob das bei der Kondensation von Benzonitril mit Chloral in Gegenwart von konz. Schwefelsäure oder Chlorsulfonsäure entstehende Produkt die Konstitution eines $\beta, \beta, \beta$-Trichlor- $\alpha, \alpha$ bis-[phenyl-carbamido]-äthans (I) oder einer $\beta, \beta, \beta$ -

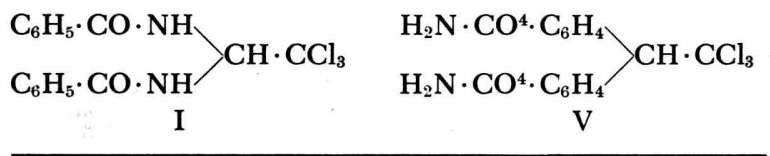<smiles>C[14CH]c1ccccc1</smiles>

II<smiles>O=C(O)CCCCC(CCCC(=O)O)C(Cl)(Cl)Cl</smiles>

III zukommt, bedürfen die Angaben jener Autoren einer Berichtigung, da die von ihnen als $\mathrm{V}$ angesprochene Verbindung eine andere Konstitution besitzt (nicht einmal mit I isomer ist) und infolgedessen zum Konstitutionsbeweis von I überhaupt nicht herangezogen werden durfte.

Die von den genannten Untersuchern ${ }^{3}$ als III, IV und $\mathrm{V}$ bezeichneten DDT-Derivate wurden auf Grund der eigenen Versuche als $\beta, \beta$-Dichlor - $\alpha, \alpha$-bis - [4carboxy-phenyl]-äthylen (VI), VI-Dichlorid (Xf) und $\beta, \beta$-Dichlor- $\alpha, \alpha$-bis-[4-carbamido-phenyl]-äthylen (XI)

1 Die I. Mitt. ist nur auszugsweise veröffentlicht worden: R. R i e m s c h n e i d e r; Zur Kenntnis der KontaktInsektizide II, 9. Beih., 1. Erg.-Bd. zur „Pharmazie“ 1950, S. 680 und 687. Referat in „The Chemical Age“ 1951.
Trichlor- $\alpha, \alpha$-bis-[4-carbamido-phenyl]-äthans (V) besitzt. Um für die durch andere Versuche bereits gestützte Annahme des Vorliegens von I einen weiteren Beweis zu haben, verglichen die genannten Autoren das „nach einer unzweifelhaften Methode synthetisierte Isomere $\mathrm{V}^{\text {“ }}$ mit dem Benzonitril-ChloralKondensationsprodukt (I), wobei sie die erwartete Nichtidentität von I mit dem aus $\beta, \beta, \beta$-Trichlor- $\alpha, \alpha$ bis-[4-methyl-phenyl]-äthan (II) über „III“ und „IV“ synthetisierten „V“ feststellten. Obgleich dem Benzonitril-Chloral-Kondensationsprodukt die Konstitution I

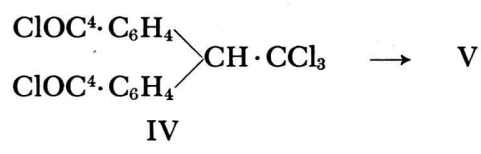

erkannt. VI vom Schmp. $276-277^{\circ}$ konnte auf verschiedenem Wege hergestellt werden, und zwar:

1. durch Oxydation von II mit Kaliumbichromat in schwefelsaurer Lösung bei Anwendung der berechneten Schwefelsäuremenge. In einigen Fällen wurde außer VI, je nach Art der Aufarbeitung, auch 4,4'Dicarboxy-diphenylmethan (IX) erhalten (Vers. 2a). Wenn unter sonst gleichen Bedingungen die Menge der Schwefelsäure auf die Hälfte reduziert wurde, entstand nicht VI, sondern $\beta, \beta, \beta$-Trichlor- $\alpha$-[4-methyl-

2 Adresse des Verfassers: Berlin-Nikolassee, Hohenzollernplatz 1 .

3 L. H a s k e l b e rg u. D. L a vi e, J. org. Chem. 14, 498 [1949].

4 Sämtliche Temperaturangaben in Celsius-Graden. 
phenyl]- $\alpha$-[4-carboxy-phenyl]-äthan (VII) vom Schmp. $160^{\circ}$ (Vers. $2 \mathrm{~b}$ );

2. in etwas besserer Ausbeute durch Oxydation von II mit Chromtrioxyd in Eisessig, je nach den Versuchsbedingungen neben VII vom Schmp. $160^{\circ}$ (Versuch 3);

3. durch Umsetzung von $\beta, \beta, \beta$-Trichlor- $\alpha, \alpha$-diphenyläthan (XII) vom Schmp. $64^{\circ}$ mit Oxalylchlorid in
Gegenwart von Aluminiumchlorid; als Nebenprodukt entstand $\beta, \beta$-Dichlor- $\alpha$-phenyl- $\alpha$-[4-carboxy-phenyl]äthylen (XIV) vom Schmp. $203^{\circ}$ (Vers. 5 a). Diese Methode liefert bei Anwendung von viel Oxalchlorid VI in sehr guter Ausbeute (Vers. 5 b).

Die Konstitution der nach den genannten Methoden aus II oder XII hergestellten VI-Säure konnte sichergestellt werden (vgl. nachstehendes Schema): Durch

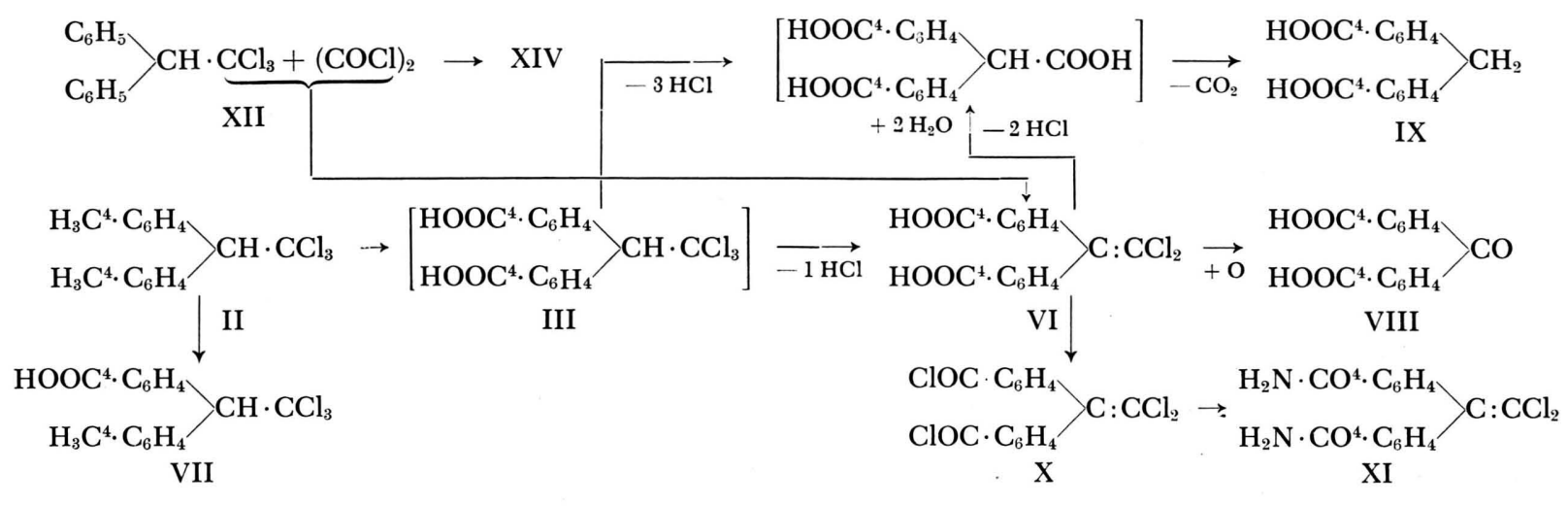

Elementaranalyse, titrimetrische Molekulargewichtsbestimmung, Überführung in den VI-Dimethylester vom Schmp. $188-198^{\circ}$ (Vers. 6), ins VI-Dichlorid (X) vom Schmp. $100^{\circ}$ und das $\beta, \beta$-Dichlor - $\alpha, \alpha$-bis - [4 carbamido-phenyl]-äthylen (XI) vom Schmp. $250^{\circ}$ (Vers. 7) sowie durch Oxydation zu 4.4'-Dicarboxybenzophenon (VIII). Eine Umlagerung der in Gegenwart von wasserfreiem Aluminiumchlorid synthetisierten VI-Säure in ein Stilbenderivat ist nicht erfolgt, da auch das mit Hilfe der Oxalylchlorid-Methode erhaltene VI-Präparat beim oxydativen Abbau VIII lieferte (Vers. 8).

Dafür, daß L. Haskelberg und D. Lavie ${ }^{3}$ nicht III, sondern ebenfalls VI in Händen hatten, spricht außer dem VI sehr ähnlichen Schmelzpunkt der von jenen Autoren hergestellten „III“-Säure 5, daß sie bei der Aufarbeitung ihrer Rohsäure mit 5proz. Natronlauge extrahierten, also unter Bedingungen arbeiteten, die eine $\mathrm{HCl}-\mathrm{Abspaltung}$ begünstigen, und daß sie für ihre „III“-Verbindung keine Chloranalyse bekanntgegeben haben. Der Einwand, daß die Schmelzpunkte von III und VI vielleicht zufällig

5 271-272 ${ }^{\circ}$, während VI nach den eigenen Versuchen bei $276-277^{\circ}$ schmilzt.

6 Versuche zur Gewinnung der reinen III-Säure aus $\beta, \beta, \beta$-Trichlor- $\alpha, \alpha$-bis-[4-amino-phenyl]-äthan über $\beta, \beta, \beta$ Trichlor- $\alpha, \alpha$-bis-[4-cyano-phenyl]-äthan werden in unserem Laboratorium z. Zt. durchgeführt. III scheint bei $\sim 245^{\circ}$ zu schmelzen und schon beim Umkristallisieren leicht in VI überzugehen. nahe beieinander liegen, läßt sich zwar zur Zeit nicht völlig entkräften, da III bisher noch nicht rein dargestellt worden ist ${ }^{6}$ und die von den genannten Autoren ${ }^{3}$ hergestellten Verbindungen zur Bestimmung des Mischschmelzpunktes nicht zur Verfügung stehen. Bei genauer Nacharbeitung der von L. H a s kelberg und D. Lavie ${ }^{3}$ angegebenen Versuche erhielt Verf. jedoch ebenfalls nur VI, das mit den nach den eigenen drei Arbeitsvorschriften synthetisierten Verbindungen keine Schmelzpunktsdepression zeigte. Entsprechend waren auch die Ergebnisse für $\mathrm{X}$ und XI ${ }^{12}$. Ähnliche Schmelzpunkte von III und VI sind überdies nicht sehr wahrscheinlich, wenn man bedenkt, daß die Schmelzpunkte vergleichbarer Säuren, wie $\beta, \beta, \beta$-Trichlor- $\alpha$-phenyl- $\alpha$-[4-carboxy-phenyl]äthan (XIII) ${ }^{1}$ und $\beta, \beta$-Dichlor- $\alpha$-phenyl- $\alpha$-[4-carboxyphenyl]-äthylen (XIV) ${ }^{1}$, etwa $60^{\circ}$ auseinanderliegen.

Die bei der Oxydation von II mit Kaliumbichromat in schwefelsaurer Lösung als Nebenprodukt erhaltene Säure VII soll nach O. F is ch e ${ }^{7}$ bei $173^{\circ}$ schmelzen. Diese Angaben ließen sich nicht bestätigen; VII, dessen Konstitution durch Elementaranalyse, Titration und Abbauversuche sichergestellt wurde, schmilzt bei $160^{\circ}$.

Sämtliche in vorliegender Mitteilung beschriebenen, bisher unbekannten Verbindungen erwiesen sich gegenüber Drosophila melanogaster M. und Musca

7 O. F i s c he r, Ber. dtsch. chem. Ges. 7, 1191 [1874]. 
domestica $\mathrm{L}$. im Petrischalen-Filmtest ${ }^{8}$ als unwirksam ${ }^{1}$. Das völlige Fehlen von kontakt-insektizider Wirkung bei den DDT-Analogen VII und XIII war zu erwarten, da durch die Anwesenheit einer COOH-Gruppe als negativer auxokontakter Substituent bedeutende Abnahme der Lipoidlöslichkeit und hoher Schmelzpunkt bedingt sind: Hochwirksame Kontakt-Insektizide mit freien $\mathrm{COOH}-G r u p p e n$ sind bisher nicht bekannt. Veresterung von VII und XIII führte ebenfalls nicht zu wirksamen Verbindungen. Da die Äthylenverbindungen, selbst der wirksamsten Vertreter der DDT-Reihe - wenn überhaupt - meist nur schwache Kontaktgiftwirkung besitzen, ist die völlige Unwirksamkeit von VI sowie der VII und XIII entsprechenden Äthylenverbindungen und ihrer Methylester selbstverständlich.*

\section{Beschreibung der Versuche ${ }^{9}$}

Vers. 1: Darstellung von $\beta, \beta, \beta-$ Trichlor$\alpha, \alpha-$ b is - [4-methy l-phenyl] - äth a n (II)

$\mathrm{Zu} 95 \mathrm{~g}$ Toluol und $75 \mathrm{~g}$ Chloral werden im Verlauf von 30 Min. unter starkem Rühren in kleinen Portionen $250 \mathrm{ccm}$ konz. Schwefelsäure gegeben, wobei mit Wasser gekühlt wird. Nach 2-stdg. Rühren und Stehenlassen über Nacht wird in ein Wasser-Eis-Gemisch gegossen und nach gründlichem Waschen mit Wasser, Filtrieren und Trocknen aus Äthylalkohol umkristallisiert. Man erhält II in weißen glänzenden Blättchen vom Schmp. $89^{\circ}$ (Ausb. $80 \%$ d. Th.).

Vers. 2: Oxydation von II mit BichromatSchwefrels äure

a) $10 \mathrm{~g}$ II werden in einer Lösung von $12 \mathrm{~g}$ konz. Schwefelsäure und $9,4 \mathrm{~g}$ Kaliumbichromat in $250 \mathrm{ccm}$ Wasser 6-7 Tage zum Sieden erhitzt. Nach dem Erkalten wird filtriert und der Filterrückstand nach mehrmaligem Waschen mit Wasser, Trocknen und Pulverisieren 10 so lange mit je $150 \mathrm{ccm}$ 10-proz. Ammoniumcarbonat-Lösung ausgekocht, bis bei Zugabe von Salzsäure zu den filtrierten und abgekühlten Salzlösungen keine Rohsäure mehr ausfällt. Gesamtmenge der Rohsäurefraktionen nach Waschen und Trocknen $\sim 1,4 \mathrm{~g}$ (Schmp. 230-290 ${ }^{\circ}$.

* Vgl. in diesem Zusammenhang die Ausführungen des Verf. auf S. 692 bis 695 der in Fußnote 1 zitierten Arbeit und Angew. Chem. A 59, 250 [1947], und A 60, 70 [1948].

8 Beschreibung der Methodik: R. Rie m s ch neider u. B. Rohrmann, Anz. Schädlingskunde 23, 148 [1950].

9 Den Herren C. B öhm (Dipl.-Arb. 1948, Berlin) und R. Martin, Leipzig, sei für ihre Mitarbeit gedankt.

10 Nach Extraktion der Säuren konnten aus diesem Oxydationsrückstand durch Extrahieren mit Äthylalkohol im Soxhlet-Apparat $50-70 \%$ nichtoxydierter Verbindungen, die infolge $\mathrm{HCl}$-Abspaltung nur zum Teil mit II identisch waren, wieder gewonnen werden. Der Anteil an $\beta, \beta$ Dichlor- $\alpha, \alpha$-bis-[4-methyl-phenyl]-äthylen vom Schmp. $92^{\circ}$ war wechselnd. Die Bildung von 4.4'-Dimethyl-benzophenon wurde nicht beobachtet.
Durch 7-maliges Umkristallisieren der ersten drei Fraktionen $(0,9 \mathrm{~g})$ aus Äthylalkohol werden $0,6 \mathrm{~g}$ VI vom Schmp. $276-277^{\circ}$ als feine glänzende Nadeln erhalten. VI ist in Äthylalkohol, Aceton und Essigsäure leichter löslich als in Benzol, Toluol oder Nitrobenzol. In Wasser ist VI sehr wenig löslich.

$$
\begin{array}{ll}
\mathrm{C}_{16} \mathrm{H}_{10} \mathrm{O}_{4} \mathrm{Cl}_{2} \text { (337). } & \text { Ber. C 57,0, H 2,98, Cl 21,1. } \\
& \text { Gef. C 57,2, H 3,10, Cl 20,8. }
\end{array}
$$

Molekulargewichtsbestimmung von VI durch Titration: $25,0 \mathrm{mg}$ VI, gelöst in 5,0 ccm Äthylalkohol, verbr. $1,58 \mathrm{ccm}$ 0,1- $n$. Natronlauge. Daraus berechnetes Mol.-Gew.: 316.

Nach Auskochen der restlichen Fraktionen der Rohsäure $(0,4 \mathrm{~g})$ mit $10 \mathrm{ccm}$ heißem Äthylalkohol hinterblieb ein schwerlöslicher, chlorfreier Rückstand von $0,3 \mathrm{~g}$, aus dem nach Umkristallisieren aus Eisessig IX erhalten wurde. IX sublimiert bei $322-323^{\circ}$.

$$
\begin{array}{ll}
\mathrm{C}_{15} \mathrm{H}_{12} \mathrm{O}_{4} \text { (256). } & \text { Ber. C 70,3, H 4,68. } \\
& \text { Gef. C 70,1, H 4,46. }
\end{array}
$$

Molekulargewichtsbestimmung von IX durch Titration: $28,2 \mathrm{mg}$ IX verbr. $2,15 \mathrm{ccm} 0,1-n$. Natronlauge. Daraus berechnetes Mol.-Gew.: 262.

Entsprechende Versuche mit doppelter bis vierfacher Menge Oxydationsmittel oder Einhaltung einer Reaktionsdauer von $320 \mathrm{Stdn}$. unter Rühren erhöhten die Ausbeute an VI nicht wesentlich. Da bei der Aufarbeitung unter milderen Bedingungen, z. B. Extrahieren des abfiltrierten Oxydationsproduktes mit Benzol (Aceton) und anschlieBender Behandlung mit 10-proz. Ammoniumcarbonat-Lösung bei $60^{\circ}$ oder mit 8-proz. Natriumbicarbonat-Lösung bei $40^{\circ}$ unter Turbinieren, nur VI gefaßt wurde, ist anzunehmen, daß IX nicht bei der Oxydation, sondern bei der oben zunächst beschriebenen Aufarbeitung von VI entsteht.

In 18-proz. Ausbeute konnte VI erhalten werden, wenn die Aufarbeitung wie folgt abgeändert wurde: Das abfiltrierte, getrocknete und zerkleinerte Reaktionsprodukt wird mit $150 \mathrm{ccm}$ Aceton digeriert und filtriert. Das Filtrat wird mit reinem Talkum zu einem Brei verührt, das Aceton weggezogen und der Rückstand pulverisiert. Die Rohsäure läßt sich daraus durch 3-maliges Behandeln mit je $200 \mathrm{ccm}$ 8-proz. Bicarbonat-Lösung bei $40^{\circ}$ gut extrahieren ('Turbinieren). VI wird aus verd. Essigsäure oder Äthylalkohol umkristallisiert.

b) $10 \mathrm{~g}$ II werden in einer Lösung von $5 \mathrm{~g}$ konz. Schwefelsäure ${ }^{11}$ und 9,4 g Kaliumbichromat in $250 \mathrm{ccm}$ Wasser 3 Tage zum Sieden erhitzt. Die Aufarbeitung des beim Abfiltrieren erhaltenen Rohproduktes erfolgt durch Extrahieren mit Benzol und Einwirkung von 8-proz. Natriumbicarbonat-Lösung bei $40^{\circ}$ oder mit AmmoniumcarbonatLösung bei $60^{\circ}$ unter Turbinieren. Die Rohsäure schmilzt bei $120-130^{\circ}$, also wesentlich niedriger als das im Vers. 2 a erhaltene Rohprodukt. Das Ammoniumsalz ist verhältnismäßig schwer "löslich und fällt in glänzenden Nadeln vom Schmp. $187^{\circ}$. Nach 7-maligem Umkristallisie-

11 Die Hälfte der theoretisch notwendigen Schwefelsäuremenge. 
ren aus wäßrigem Äthylalkohol erhält man VII vom Schmp. $160^{\circ}$ in glänzenden weißen Blättchen.

$$
\mathrm{C}_{16} \mathrm{H}_{13} \mathrm{O}_{2} \mathrm{Cl}_{3}(343,6) \text {. Ber. Cl 31,0. Gef. Cl 30,7. }
$$

Molekulargewichtsbestimmung von VII durch Titration: $20,0 \mathrm{mg}$ VII verbr. $0,6 \mathrm{ccm} 0,1-n$. Natronlauge. Daraus berechnetes Mol.-Gew.: 333.

$$
\begin{aligned}
& \text { Vers. 3: Oxydation von II } \\
& \text { mit Chromtrioxyd-Eisessig }
\end{aligned}
$$

$10 \mathrm{~g}$ II in $200 \mathrm{ccm}$ Eisessig werden nach Zugabe von $6,4 \mathrm{~g} \mathrm{CrO}_{3}$ im Ölbad zum schwachen Sieden erhitzt. Der Verbrauch des Oxydationsmittels wird durch jodometrische Titration stündlich entnommener Proben verfolgt. Nach $5 \mathrm{Stdn}$. ist die für eine Methylgruppe berechnete Menge Chromtrioxyd verbraucht. Nach dem Erkalten und Abdestillieren der Hauptmenge des Eisessigs im Vakuum wird der Rückstand mit Wasser versetzt, filtriert, gewaschen und getrocknet. Die Abtrennung der Rohsäure erfolgt, wie im Vers. 2 a beschrieben, durch Extrahieren mit Aceton, Verrühren mit Talkum und Einwirkung von 8-proz. Bicarbonat-Lösung auf das getrocknete, pulverisierte Produkt unter Turbinieren. Ausbeute der Rohsäure vom Schmp. $120-180^{\circ} 2,1$ g. Durch fraktionierte Kristallisation aus $30 \mathrm{ccm}$ Benzol, in welchem VI schwerer löslich ist als VII, können die beiden Säuren getrennt werden. Das Umkristallisieren von VI und VII erfolgt aus wäßrigem Äthylalkohol.

Bei Erhöhung der Menge des Oxydationsmittels auf das Doppelte $(12,8 \mathrm{~g})$ und einer Oxydationsdauer von 12 Stdn. steigt die Ausbeute an VI etwas an, während VII nur noch Nebenprodukt ist. Bei 18-stdg. Versuchsdauer wurde VI als einzige Säure gefaßt.

Vers. 4: Darstellung von $\beta, \beta, \beta-\mathrm{Trichlor}-$ diphenyl-äthan (XII)

$\mathrm{Zu} 160 \mathrm{~g} \mathrm{Benzol}$ und $150 \mathrm{~g} \mathrm{Chloral}$ werden unter Rühren aus einem Tropftrichter portionsweise $500 \mathrm{ccm}$ konz. Schwefelsäure gegeben, wobei mit Wasser gekühlt wird. Versuchsdauer $4 \mathrm{Stdn}$. Aufarbeitung wie im Vers. 1. Man erhält XII in weißen glänzenden Blättchen vom Schmp. $64^{\circ}$ (Ausb. 85\% d. Th.).

\section{Vers. 5: Einwirkung von Oxalylchlorid} a uf XII

a) Zu $10 \mathrm{~g}$ XII und $16 \mathrm{~g} \mathrm{Oxaly} \mathrm{l} \mathrm{chlorid} \mathrm{in} 75 \mathrm{ccm}$ trockenem Schwefelkohlenstoff (dest. über Aluminiumchlorid) werden bei Zimmertemperatur unter starkem Rühren im Verlauf von $2 \mathrm{Stdn}$. portionsweise $5 \mathrm{~g}$ pulverisiertes wasserfreies $\mathrm{Al} \mathrm{u} \mathrm{mini} \mathrm{m} \mathrm{chlorid}$ gegeben. Nach Zusatz von weiteren $75 \mathrm{ccm} \mathrm{CS}_{2}$ wird noch $2 \mathrm{Stdn}$. gerührt, wobei Dunkelfärbung und schwache HCl-Entwicklung zu beobachten ist, und nach 24-stdg. Stehenlassen in eisgekühlte Salzsäure 'gegossen und getrennt. Nach Abdestillieren des $\mathrm{CS}_{2}$, Filtration und Waschen mit Wasser wird der Rückstand mehrmals mit je $50 \mathrm{ccm}$ warmem Aceton ausgezogen. Die filtrierte Lösung wird mit Talkum verrührt, wobei das Aceton verdunstet. Das pulverisierte Produkt wird 3-mal mit je $150 \mathrm{ccm}$ 8-proz. Natriumcarbonat-Lösung bei Zimmertemperatur 20 Min. turbiniert. Ausbeute an Rohsäuren 3,0 g. Die mit Wasser gewaschene und getrocknete Rohsäure wird bei $40^{\circ}$ mit $15 \mathrm{ccm}$ Benzol behandelt und filtriert. Nach 6-maligem Umkristallisieren des Filterrückstandes aus wäßrigem Äthylalkohol wird VI vom Schmp. 275-276 ${ }^{\circ}$ erhalten. Der aus dem Filtrat der Benzollösungen nach Abdampfen des Benzols bleibende Rückstand wird in Alkohol gelöst und durch Zusatz von Wasser XIV gefällt. Nach 4-maligem Umkristallisieren erhält man XIV in glänzenden Nadeln vom Schmp. $203^{\circ}$. XIV ist in Alkohol, Essigsäure, Äther und Aceton leichter löslich als in Toluol.

$$
\begin{array}{r}
\mathrm{C}_{15} \mathrm{H}_{10} \mathrm{O}_{2} \mathrm{Cl}_{2}(293,1) . \\
\text { Ber. C 61,4, H 3,41, Cl 24,2. } \\
\text { Gef. C 61,5, H 3,54, Cl 24,3. }
\end{array}
$$

Molekulargewichtsbestimmung durch Titration: 25,0 mg XIV verbr. $0,79 \mathrm{ccm}$ 0,1- $n$. Natronlauge. Daraus berechnetes Mol.-Gew.: 316.

b) Bei der Umsetzung von $10 \mathrm{~g}$ XII in $80 \mathrm{ccm} \mathrm{CS}_{2}, 30 \mathrm{~g}$ $\mathrm{Oxalylchlorid}$ und $10 \mathrm{~g}$ Aluminiumchlorid bei $0^{\circ}$ läßt sich eine wesentliche Steigerung der Ausbeute an VI erzielen. Es wurden 9,4 g Rohsäuren (80-90\%) erhalten, aus denen VI und XIV wie im Vers. 5 a abgetrennt werden konnten. Die Säure VI ist Hauptprodukt.

\section{Vers. 6: Veresterung der Säuren VI und XIV}

Eine Lösung von $300 \mathrm{mg}$ VI in $20 \mathrm{ccm}$ Methylalkohol wird bei $0^{\circ}$ mit $\mathrm{HCl}$ gesättigt, $30 \mathrm{Min}$. erhitzt und nach dem Erkalten nochmals mit $\mathrm{HCl}$ gesättigt. Da der Ester schwerer löslich ist als VI, kristallisiert er bereits beim Erkalten z. Tl. aus. Nach Abdestillieren des Methanols im Vakuum wird der Rückstand aus $8 \mathrm{ccm} 80$-proz. heißer Essigsäure umkristallisiert. Der VI - D i m e th y le s t e r schmilzt bei $188-189^{\circ}$.

$$
\begin{array}{ll}
\mathrm{C}_{18} \mathrm{H}_{14} \mathrm{O}_{4} \mathrm{Cl}_{2}(365,2) . & \text { Ber. C 59,2, H 3,86, Cl 19,4. } \\
& \text { Gef. C 59,7, H 4,15, Cl 20,2. }
\end{array}
$$

Der analog hergestellte XIV - M e t h y le s t e r schmilzt bei $64,5-65^{\circ}$.

$$
\begin{array}{ll}
\mathrm{C}_{16} \mathrm{H}_{12} \mathrm{O}_{2} \mathrm{Cl}_{2}(307,2) . & \text { Ber. C 62,8, H 3,91, Cl 23,2. } \\
& \text { Gef. C 63,1, H 4,46, Cl 23,2. }
\end{array}
$$

Vers. 7: Darstellung des VI-Dichlorids (X) und VI - D i a mids (XI)

$2 \mathrm{~g}$ VI und $10 \mathrm{~g} \mathrm{Th}$ i o n y l c h lorid (XV) werden im Wasserbad 2 Stdn. unter Rückfluß erhitzt. Das nach dem Abdestillieren des überschüssigen $\mathrm{XV}$ im Vakuum von Kristallen durchsetzte Öl wird mit der gleichen Menge Äther versetzt und geteilt. X kristallisiert aus der Ätherlösung nach kurzer Zeit in weichen filzigen Nadeln, die mit Petroläther gewaschen werden, aus und schmilzt bei $100^{\circ}$. Die andere Hälfte der ätherischen Lösung wird langsam zu der gleichen Menge einer 15-proz. wäßrigen Ammoniaklösung gegeben, wobei sich das VI - D i a m i d (XI) abscheidet; das abfiltrierte, mit Wasser gewaschene, 
getrocknete und aus Eisessig umkristallisierte XI schmilzt bei $250^{\circ} 12$. Ausb. $1,1 \mathrm{~g}$ (gelbliche glänzende Prismen).

$$
\begin{gathered}
\mathrm{C}_{16} \mathrm{H}_{12} \mathrm{O}_{2} \mathrm{~N}_{2} \mathrm{Cl}_{2}(335,2) \text {. Ber. Cl 21,2. Gef. Cl 21,3. } \\
\text { Vers. 8: Oxy d a tion von VI } \\
\text { z u 4.4' - Dic a r box y - benzophenon (VIII) }
\end{gathered}
$$

$\mathrm{Zu}$ einer siedenden Lösung von $1,5 \mathrm{~g}$ VI in $8 \mathrm{ccm}$ Eisessig wird im Verlauf von $1 / 2$ Stde. $1 \mathrm{~g}$ Chromtrioxyd in $4 \mathrm{ccm}$ Eisessig gegeben und 2 Stdn. erhitzt. Nach dem Erkalten wird in $150 \mathrm{ccm}$ Eiswasser gegossen und ab- filtriert, mit Wasser gewaschen und getrocknet. Nach Umkristallisieren aus Eisessig erhält man VIII in feinen weißen Kristallen vom Schmp. $>362^{\circ}$ (sublimierend). Ausb. $1 \mathrm{~g}$.

Durch Erhitzen mit Thionylchlorid werden 0,2 g VIII in das VIII - D i c hlor id übergeführt. Aus Toluol erhält man das VIII-Dichlorid in weißen Kristallen vom Schmp. $133^{\circ}$.

12 L. H a s k elberg u. D. Lavi e ${ }^{3}$ geben für diese von ihnen irrtümlicherweise als $\mathrm{V}$ angesehene Verbindung den Schmp. $227^{\circ}$ an.

\title{
Untersuchungen zur Toxizität der $p$-Aminosalicylsäure und ihrer Derivate
}

\author{
Von Paul P. Koelzer und Johannes Giesen \\ Aus der Kinderklinik der Medizinischen Akademie Düsseldorf (Direktor: Prof. Dr. F. Goebel $\nmid$ ) \\ (Z. Naturforschg. 6 b, 183-190 [1951]; eingegangen am 28. Februar 1951)
}

Bei einer experimentellen Untersuchung der Toxizität der PAS und ihrer Derivate an verschiedenen Laboratoriumstieren wurden folgende Ergebnisse erzielt:

1. Die Toxizität einer Einzeldosis an PAS in Form der freien Säure bzw. ihrer Salze ist sehr gering, wenn man nur die akute Vergiftung als Kriterium heranzieht.

2. Bei der Dauerbehandlung mit PAS-Säure bzw. PAS-Natrium ist es erforderlich, in der Tagesdosis unter einer bestimmten Maximaldosis, die etwa $1 / 3$ der Dosis maxima tolerata (Einzelgabe) umfaßt, zu bleiben, wenn die Versuchstiere ohne Schaden überleben sollen. Bei Dauerversuchen mit Mäusen muß von einer Anwendung der PAS-Säure abgesehen werden, da deren Wasserunlöslichkeit und schwere Resorbierbarkeit zusätzliche Schäden hervorruft, die die Feststellung einer wirklichen, pharmakologischen Dauerverträglichkeit ausschließen.

3. Die histologische Untersuchung von Ratten, die einer langdauernden Behandlung mit PAS unterzogen worden waren, ergab keinerlei pathologische Veränderungen in den einzelnen Organen.

4. Die im Körper während einer PAS-Therapie entstehende $N$-Acetyl-PAS besaß, peroral verabreicht, eine bedeutend geringere Toxizität als PAS selbst.

5. Dagegen zeigte $m$-Aminophenol, das in erhitzten PAS-Na-Lösungen leicht entsteht, die 20-fache Giftigkeit der PAS.

6. Bei der Untersuchung der Zersetzungserscheinungen wäßriger PAS-Na-Lösungen konnte festgestellt werden, daß die Verfärbung der Lösungen nicht mit der Zunahme der Toxizität durch $m$-Aminophenol-Bildung parallel geht. Braun verfärbte Lösungen sind nur wenig tcxischer, erhitzte Teile dagegen, auch wenn sie farblos bleiben, meist sehr toxisch.

7. Weitere Toxizitätsangaben verschiedener Nitro- und Amino-salicylsäuren sowie einiger Alkylester der PAS werden angeführt. Basische Ester von der Art des Novocains besaßen eine Toxizität, die der des Novocains ähnlich ist, auch zeigten diese Ester (S 557 und SK 8) die gleiche anästhesierende Kraft wie Novocain.

$\mathrm{V}$ eröffentlichungen über die klinische Anwendung der $p$-Amino-salicylsäure (PAS) bei der menschlichen Tuberkulose liegen bereits in großer Zahl vor und nehmen noch dauernd weiter zu. Dagegen scheint das Material über die Eigenschaften der PAS als Fharmakologische Substanz, vor allem über die Untersuchung ihrer Toxizität, soweit wir feststellen konnten, sehr wenig umfangreich. Wir glauben daher eine Lücke zu schließen, wenn wir in vorliegendem Bericht die Ergebnisse unserer Toxizitätsuntersuchungen, die zur Zeit der Einführung der PAS in Deutschland von uns gesammelt wurden, der Öffentlichkeit vorlegen. Der Übersichtlichkeit halber soll das Material, das neben der PAS selbst noch mehrere ihrer Derivate und Abbauprodukte berücksichtigt, in einzelnen Punkten zusammengefaßt dargestellt werden.

\section{Toxizität der PAS und ihrer Salze}

1. Die Toxizität einer Einzelgabe (LD $\left.D_{z a}\right)$

Untersucht wurden die freie PAS-Säure, ihr Natriumund Calcium-Salz. Wegen ihrer Unlöslichkeit mußte die freie Säure, in Gummischleim bzw. vegetabilischem Öl suspendiert, verabreicht werden, die Salze in Form: konzentrierter wäßriger Lösungen. Die in mehrfach 